\title{
Several Classes of BCK-algebras and their Properties
}

\author{
Tao Sun \\ Qingdao University of Science \\ and Technology \\ China
}

\author{
Dahai $\mathrm{Hu}$ \\ Qingdao University of Science \\ and Technology \\ China
}

\author{
Xiquan Liang \\ Qingdao University of Science \\ and Technology \\ China
}

\begin{abstract}
Summary. In this article the general theory of Commutative BCKalgebras and BCI-algebras and several classes of BCK-algebras are given according to $[2]$.
\end{abstract}

MML identifier: BCIALG_3, version: 7.8.05 4.89.993

The articles [3] and [1] provide the notation and terminology for this paper.

1. The Basics of General Theory of Commutative BCK-Algebras

Let $I_{1}$ be a non empty BCI structure with 0 . We say that $I_{1}$ is commutative if and only if:

(Def. 1) For all elements $x, y$ of $I_{1}$ holds $x \backslash(x \backslash y)=y \backslash(y \backslash x)$.

Let us observe that BCI-EXAMPLE is commutative.

Let us note that there exists a BCK-algebra which is commutative.

In the sequel $X$ denotes a BCK-algebra and $I_{1}$ denotes a non empty subset of $X$.

We now state a number of propositions:

(1) $X$ is a commutative BCK-algebra iff for all elements $x, y$ of $X$ holds $x \backslash(x \backslash y) \leq y \backslash(y \backslash x)$. 
(2) For every BCK-algebra $X$ and for all elements $x, y$ of $X$ holds $x \backslash(x \backslash y) \leq$ $y$ and $x \backslash(x \backslash y) \leq x$.

(3) $X$ is a commutative BCK-algebra iff for all elements $x, y$ of $X$ holds $x \backslash y=x \backslash(y \backslash(y \backslash x))$.

(4) $X$ is a commutative BCK-algebra iff for all elements $x, y$ of $X$ holds $x \backslash(x \backslash y)=y \backslash(y \backslash(x \backslash(x \backslash y)))$.

(5) $X$ is a commutative BCK-algebra iff for all elements $x, y$ of $X$ such that $x \leq y$ holds $x=y \backslash(y \backslash x)$.

(6) Let $X$ be a non empty BCI structure with 0 . Then $X$ is a commutative BCK-algebra if and only if for all elements $x, y, z$ of $X$ holds $x \backslash\left(0_{X} \backslash y\right)=x$ and $(x \backslash z) \backslash(x \backslash y)=y \backslash z \backslash(y \backslash x)$.

(7) If $X$ is a commutative BCK-algebra, then for all elements $x, y$ of $X$ such that $x \backslash y=x$ holds $y \backslash x=y$.

(8) If $X$ is a commutative BCK-algebra, then for all elements $x, y, a$ of $X$ such that $y \leq a$ holds $a \backslash x \backslash(a \backslash y)=y \backslash x$.

(9) If $X$ is a commutative BCK-algebra, then for all elements $x, y$ of $X$ holds $x \backslash y=x$ iff $y \backslash(y \backslash x)=0_{X}$.

(10) If $X$ is a commutative BCK-algebra, then for all elements $x, y$ of $X$ holds $x \backslash(y \backslash(y \backslash x))=x \backslash y$ and $x \backslash y \backslash(x \backslash y \backslash x)=x \backslash y$.

(11) Suppose $X$ is a commutative BCK-algebra. Let $x, y, a$ be elements of $X$. If $x \leq a$, then $(a \backslash y) \backslash(a \backslash y \backslash(a \backslash x))=a \backslash y \backslash(x \backslash y)$.

Let $X$ be a BCI-algebra and let $a$ be an element of $X$. We say that $a$ is greatest if and only if:

(Def. 2) For every element $x$ of $X$ holds $x \backslash a=0_{X}$.

We say that $a$ is positive if and only if:

(Def. 3) $0_{X} \backslash a=0_{X}$.

\section{The Basics of General Theory of Commutative BCI-Algebras}

Let $I_{1}$ be a BCI-algebra. We say that $I_{1}$ is BCI-commutative if and only if: (Def. 4) For all elements $x, y$ of $I_{1}$ such that $x \backslash y=0_{\left(I_{1}\right)}$ holds $x=y \backslash(y \backslash x)$.

We say that $I_{1}$ is BCI-weakly-commutative if and only if:

(Def. 5) For all elements $x, y$ of $I_{1}$ holds $(x \backslash(x \backslash y)) \backslash\left(0_{\left(I_{1}\right)} \backslash(x \backslash y)\right)=y \backslash(y \backslash x)$.

One can check that BCI-EXAMPLE is BCI-commutative and BCI-weaklycommutative.

Let us note that there exists a BCI-algebra which is BCI-commutative and BCI-weakly-commutative.

The following propositions are true: 
(12) For every BCI-algebra $X$ such that there exists an element of $X$ which is greatest holds $X$ is a BCK-algebra.

(13) Let $X$ be a BCI-algebra. Suppose $X$ is $p$-semisimple. Then $X$ is BCIcommutative and BCI-weakly-commutative.

(14) Every commutative BCK-algebra is a BCI-commutative BCI-algebra and a BCI-weakly-commutative BCI-algebra.

(15) If $X$ is a BCI-weakly-commutative BCI-algebra, then $X$ is BCIcommutative.

(16) Let $X$ be a BCI-algebra. Then $X$ is BCI-commutative if and only if for all elements $x, y$ of $X$ holds $x \backslash(x \backslash y)=y \backslash(y \backslash(x \backslash(x \backslash y)))$.

(17) Let $X$ be a BCI-algebra. Then $X$ is BCI-commutative if and only if for all elements $x, y$ of $X$ holds $(x \backslash(x \backslash y)) \backslash(y \backslash(y \backslash x))=0_{X} \backslash(x \backslash y)$.

(18) Let $X$ be a BCI-algebra. Then $X$ is BCI-commutative if and only if for every element $a$ of AtomSet $X$ and for all elements $x, y$ of BranchV $a$ holds $x \backslash(x \backslash y)=y \backslash(y \backslash x)$.

(19) Let $X$ be a non empty BCI structure with 0 . Then $X$ is a BCIcommutative BCI-algebra if and only if for all elements $x, y, z$ of $X$ holds $x \backslash y \backslash(x \backslash z) \backslash(z \backslash y)=0_{X}$ and $x \backslash 0_{X}=x$ and $x \backslash(x \backslash y)=y \backslash(y \backslash(x \backslash(x \backslash y)))$.

(20) Let $X$ be a BCI-algebra. Then $X$ is BCI-commutative if and only if for all elements $x, y, z$ of $X$ such that $x \leq z$ and $z \backslash y \leq z \backslash x$ holds $x \leq y$.

(21) Let $X$ be a BCI-algebra. Then $X$ is BCI-commutative if and only if for all elements $x, y, z$ of $X$ such that $x \leq y$ and $x \leq z$ holds $x \leq y \backslash(y \backslash z)$.

\section{Bounded BCK-ALGEBRAS}

Let $I_{1}$ be a BCK-algebra. We say that $I_{1}$ is bounded if and only if:

(Def. 6) There exists an element of $I_{1}$ which is greatest.

Let us note that BCI-EXAMPLE is bounded.

One can verify that there exists a BCK-algebra which is bounded and commutative.

Let $I_{1}$ be a bounded BCK-algebra. We say that $I_{1}$ is involutory if and only if:

(Def. 7) For every element $a$ of $I_{1}$ such that $a$ is greatest and for every element $x$ of $I_{1}$ holds $a \backslash(a \backslash x)=x$.

Next we state three propositions:

(22) Let $X$ be a bounded BCK-algebra. Then $X$ is involutory if and only if for every element $a$ of $X$ such that $a$ is greatest and for all elements $x, y$ of $X$ holds $x \backslash y=a \backslash y \backslash(a \backslash x)$. 
(23) Let $X$ be a bounded BCK-algebra. Then $X$ is involutory if and only if for every element $a$ of $X$ such that $a$ is greatest and for all elements $x, y$ of $X$ holds $x \backslash(a \backslash y)=y \backslash(a \backslash x)$.

(24) Let $X$ be a bounded BCK-algebra. Then $X$ is involutory if and only if for every element $a$ of $X$ such that $a$ is greatest and for all elements $x, y$ of $X$ such that $x \leq a \backslash y$ holds $y \leq a \backslash x$.

Let $I_{1}$ be a BCK-algebra and let $a$ be an element of $I_{1}$. We say that $a$ is Iseki if and only if:

(Def. 8) For every element $x$ of $I_{1}$ holds $x \backslash a=0_{\left(I_{1}\right)}$ and $a \backslash x=a$.

Let $I_{1}$ be a BCK-algebra. We say that $I_{1}$ is Iseki-extension if and only if:

(Def. 9) There exists an element of $I_{1}$ which is Iseki.

Let us observe that BCI-EXAMPLE is Iseki-extension.

Let $X$ be a BCK-algebra. A non empty subset of $X$ is said to be a commutative-ideal of $X$ if:

(Def. 10) $0_{X} \in$ it and for all elements $x, y, z$ of $X$ such that $x \backslash y \backslash z \in$ it and $z \in$ it holds $x \backslash(y \backslash(y \backslash x)) \in$ it.

The following three propositions are true:

(25) If $I_{1}$ is a commutative-ideal of $X$, then for all elements $x, y$ of $X$ such that $x \backslash y \in I_{1}$ holds $x \backslash(y \backslash(y \backslash x)) \in I_{1}$.

(26) For every BCK-algebra $X$ such that $I_{1}$ is a commutative-ideal of $X$ holds $I_{1}$ is an ideal of $X$.

(27) If $I_{1}$ is a commutative-ideal of $X$, then for all elements $x, y$ of $X$ such that $x \backslash(x \backslash y) \in I_{1}$ holds $y \backslash(y \backslash x) \backslash(x \backslash y) \in I_{1}$.

\section{Implicative and Positive-Implicative BCK-Algebras}

Let $I_{1}$ be a BCK-algebra. We say that $I_{1}$ is BCK-positive-implicative if and only if:

(Def. 11) For all elements $x, y, z$ of $I_{1}$ holds $(x \backslash y) \backslash z=x \backslash z \backslash(y \backslash z)$.

We say that $I_{1}$ is BCK-implicative if and only if:

(Def. 12) For all elements $x, y$ of $I_{1}$ holds $x \backslash(y \backslash x)=x$.

Let us observe that BCI-EXAMPLE is BCK-positive-implicative and BCKimplicative.

Let us mention that there exists a BCK-algebra which is Iseki-extension, BCK-positive-implicative, BCK-implicative, bounded, and commutative.

The following propositions are true:

(28) $X$ is a BCK-positive-implicative BCK-algebra iff for all elements $x, y$ of $X$ holds $x \backslash y=x \backslash y \backslash y$. 
(29) $X$ is a BCK-positive-implicative BCK-algebra if and only if for all elements $x, y$ of $X$ holds $(x \backslash(x \backslash y)) \backslash(y \backslash x)=x \backslash(x \backslash(y \backslash(y \backslash x)))$.

(30) $X$ is a BCK-positive-implicative BCK-algebra iff for all elements $x, y$ of $X$ holds $x \backslash y=x \backslash y \backslash(x \backslash(x \backslash y))$.

(31) $X$ is a BCK-positive-implicative BCK-algebra if and only if for all elements $x, y, z$ of $X$ holds $x \backslash z \backslash(y \backslash z) \leq(x \backslash y) \backslash z$.

(32) $X$ is a BCK-positive-implicative BCK-algebra iff for all elements $x, y$ of $X$ holds $x \backslash y \leq x \backslash y \backslash y$.

(33) $X$ is a BCK-positive-implicative BCK-algebra if and only if for all elements $x, y$ of $X$ holds $x \backslash(x \backslash(y \backslash(y \backslash x))) \leq(x \backslash(x \backslash y)) \backslash(y \backslash x)$.

(34) $X$ is a BCK-implicative BCK-algebra if and only if $X$ is a commutative BCK-algebra and a BCK-positive-implicative BCK-algebra.

(35) $X$ is a BCK-implicative BCK-algebra iff for all elements $x, y$ of $X$ holds $(x \backslash(x \backslash y)) \backslash(x \backslash y)=y \backslash(y \backslash x)$.

(36) Let $X$ be a non empty BCI structure with 0 . Then $X$ is a BCKimplicative BCK-algebra if and only if for all elements $x, y, z$ of $X$ holds $x \backslash\left(0_{X} \backslash y\right)=x$ and $(x \backslash z) \backslash(x \backslash y)=y \backslash z \backslash(y \backslash x) \backslash(x \backslash y)$.

(37) Let $X$ be a bounded BCK-algebra and $a$ be an element of $X$. Suppose $a$ is greatest. Then $X$ is BCK-implicative if and only if $X$ is involutory and BCK-positive-implicative.

(38) $X$ is a BCK-implicative BCK-algebra iff for all elements $x, y$ of $X$ holds $x \backslash(x \backslash(y \backslash x))=0_{X}$.

(39) $X$ is a BCK-implicative BCK-algebra iff for all elements $x, y$ of $X$ holds $(x \backslash(x \backslash y)) \backslash(x \backslash y)=y \backslash(y \backslash(x \backslash(x \backslash y)))$.

(40) $X$ is a BCK-implicative BCK-algebra iff for all elements $x, y, z$ of $X$ holds $(x \backslash z) \backslash(x \backslash y)=y \backslash z \backslash(y \backslash x \backslash z)$.

(41) $X$ is a BCK-implicative BCK-algebra iff for all elements $x, y, z$ of $X$ holds $x \backslash(x \backslash(y \backslash z))=(y \backslash z) \backslash(y \backslash z \backslash(x \backslash z))$.

(42) $X$ is a BCK-implicative BCK-algebra iff for all elements $x, y$ of $X$ holds $x \backslash(x \backslash y)=(y \backslash(y \backslash x)) \backslash(x \backslash y)$.

(43) Let $X$ be a bounded commutative BCK-algebra and $a$ be an element of $X$. Suppose $a$ is greatest. Then $X$ is BCK-implicative if and only if for every element $x$ of $X$ holds $a \backslash x \backslash(a \backslash x \backslash x)=0_{X}$.

(44) Let $X$ be a bounded commutative BCK-algebra and $a$ be an element of $X$. Suppose $a$ is greatest. Then $X$ is BCK-implicative if and only if for every element $x$ of $X$ holds $x \backslash(a \backslash x)=x$.

(45) Let $X$ be a bounded commutative BCK-algebra and $a$ be an element of $X$. Suppose $a$ is greatest. Then $X$ is BCK-implicative if and only if for every element $x$ of $X$ holds $a \backslash x \backslash x=a \backslash x$. 
(46) Let $X$ be a bounded commutative BCK-algebra and $a$ be an element of $X$. Suppose $a$ is greatest. Then $X$ is BCK-implicative if and only if for all elements $x, y$ of $X$ holds $a \backslash y \backslash(a \backslash y \backslash x)=x \backslash y$.

(47) Let $X$ be a bounded commutative BCK-algebra and $a$ be an element of $X$. Suppose $a$ is greatest. Then $X$ is BCK-implicative if and only if for all elements $x, y$ of $X$ holds $y \backslash(y \backslash x)=x \backslash(a \backslash y)$.

(48) Let $X$ be a bounded commutative BCK-algebra and $a$ be an element of $X$. Suppose $a$ is greatest. Then $X$ is BCK-implicative if and only if for all elements $x, y, z$ of $X$ holds $(x \backslash(y \backslash z)) \backslash(x \backslash y) \leq x \backslash(a \backslash z)$.

\section{REFERENCES}

[1] Yuzhong Ding. Several classes of BCI-algebras and their properties. Formalized Mathematics, 15(1):1-9, 2007.

[2] Jie Meng and YoungLin Liu. An Introduction to BCI-algebras. Shaanxi Scientific and Technological Press, 2001.

[3] Zinaida Trybulec. Properties of subsets. Formalized Mathematics, 1(1):67-71, 1990.

Received September 19, 2007 